\title{
Numerical Simulation of Waveform Adjustment in high G Accelerometer Calibration System Using Hopkinson Bar
}

\author{
ZHAO Xing-liang ${ }^{1}$, LI Xin-liang ${ }^{1}$, XU Peng $^{2}$, ZHANG Da-zhi ${ }^{1}$ \\ ${ }^{1}$ Science and Technology on Metrology and Calibration Laboratory, Changcheng Institute of \\ Metrology and Measurement, Beijing100095, China; \\ ${ }^{2}$ School of Science, North University of China, Taiyuan030051, Shanxi, China \\ hunter2011@foxmail.com
}

Keywords: Hopkinson bar, Acceleration, Stress wave, Waveform adjustment.

\begin{abstract}
High-G accelerometer impact excitation using Hopkinson pressure bar was introduced. The impact excitation course was simulated on ANSYS/LS-DYNA using Lagrange's method and Johnson-Cook constitutive model. The effects of projectile nose shape, nose length, and adjustment pad material on acceleration waveforms were studied. The simulated results are in good agreement with the experimental results. The results show that projectiles with small nose taper or with large nose length will impact and produce acceleration waveform pulses with long rising time, low amplitude, and large pulse width. For adjustment pad material with small yield stress, the pulses of corresponding acceleration waveforms have long rise time, low amplitude, and large pulse width.

Along with the rapid development of silicon microtechnology, high- $\mathrm{G}$ accelerometers as an important measuring element are widely applied in load measurement during high-speed impacting. In particular, high-G accelerometers are used in study of penetration, anti-penetration, and target panel goal characteristics in the field of weapons (e.g. measurement of deceleration for armor piercing projectiles during penetration and breakthrough). High-G acceleration transducers are also widely used in civil industry, such as in anti-crash design of civil aviation aircraft, and in measurement of overload produced when plane structure drops on the ground. A high-G accelerometer's sensitivity coefficient directly affects its measurement accuracy. After repeated use, its sensitivity coefficient will change because of overload and thus it should be frequently calibrated.

In calibration of high-G accelerometers, Hopkinson bar is commonly used as a loading means, since it has high repeatability and convenient operation, and its acceleration amplitude can reach $2 \times 10^{5} \mathrm{~g}^{[1]-[3]}$. Moreover, corresponding adjustment measures are needed to endow the accelerometer's excitation signals with certain waveforms. Forrestal et al. ${ }^{[2]}$ used a $0.254-\mathrm{m}-$ long aluminum bar with flat ends to coaxially impact a 1.829-m-long aluminum input bar, and put an organic glass or copper pad between the two bars to adjust the accelerometer's output waveforms; however, the resulting waveforms were closer to rectangle and largely different from the semi-sine signals that are commonly used for calibration ${ }^{[4]}$. Li et al. ${ }^{[3]}$ used cone-shaped projectiles to coaxially impact the pad stuck to the other end of the calibration bar, and the accelerometer installed on the other end produced approximately semi-sine signals. Zhang et al. ${ }^{[5]}$ adjusted the rising edge of input waveforms from a split hopkinson pressure bar (SHPB) by changing the projectiles' shape and length. Deng et al. ${ }^{[6]}$ designed a stress waveform adjustment installation by adding a copper waveform shaper on the Hopkinson bar.

Based on the above research, in this paper, we used a display power finite element program ANSYS/LS-DYNA, and built a finite element model using high-G accelerometer impact excitation on a Hopkinson bar installation. Then this process was numerically simulated. We calculated the acceleration-time waveforms by changing nose shape, nose length, and adjustment pad materials. The simulated results were compared with the experimental results. Then the effects of nose shape, nose length, and adjusting pad material on high-G accelerometer's impact excitation waveforms were studied.
\end{abstract}




\section{Experimental principles}

Sketch of experiment installation is showed in Fig. 1. The anvil body has one free end, while the other end is closely pulled via a vacuum clamp and a coupling agent into one end of the Hopkinson bar. The anvil body's lateral face is stuck with reflection grating along the axial direction. The Hopkinson bar's other end is also closely pulled into the adjustment pad via a vacuum clamp and a coupling agent. The adjustment pad, Hopkinson bar and the anvil body have the same diameter, and the anvil body and Hopkinson bar are made of the same material. During tests, the air gun shoots a projectile with certain nose shape, which coaxially impacts the adjustment pad and produces an approximately semi-sine pressure stress wave in the bar ${ }^{[3]}$, which is propagated along the pressure bar. Because the Hopkinson bar has a large length-diameter ratio, when the stress wave is propagated in the bar, the wave dispersion effect is not considered. Since the pressure bar and the anvil body are made of the same material, the pressure stress wave will almost not reflect when passing their interface. The pressure stress wave will be reflected to tensile wave when arriving at the anvil body's free end; the incident compressive wave and the reflected tensile wave will be overlapped; when the resultant force at the interface of the pressure bar and the anvil body is dominated by static tension, the anvil body will fly away and start accelerated motion. At this point, the grating's motion, through a differential laser interferometer, will produce frequency shift signals, which are collected by a computer. Frequency demodulation and differential of this frequency-modulated signal will produce the anvil body's acceleration excitation signals ${ }^{[7]}$, which can be used as reference signals in absolute calibration of high- $G$ accelerometer laser interference ${ }^{[4]}$.

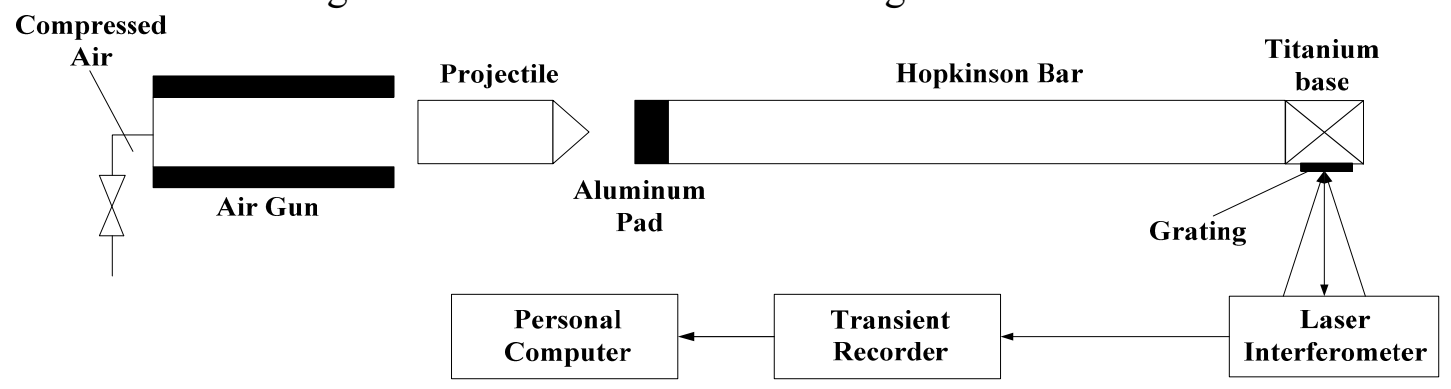

Fig.1 Sketch of experiment equipment

\section{Numerical simulation method and model}

Lagrange's method. The impact excitation course of high-G accelerometer calibration system using a Hopkinson bar is a high-speed collision process, which can be described by finite element methods such as Lagrange's method, Euler's method, and arbitrary Lagrange-Euler method (ALE). Euler's method is most widely used in fluid mechanics but rarely in solid mechanics. ALE is usually used in fluid-solid coupling, but its theories and algorithms are very complex. Moreover, models built with ALE contain huger unit data and larger amount of calculation than other algorithms. Lagrange's method is the most mature and convenient method for description of solid collision behaviors.

Lagrange's method is based on physical coordinates, and the grids it describes will be partitioned like "carving" into the structure to be analyzed. Namely, Lagrange's method will describe the grids and structure as an integrity, and the finite element nodes are substance points. With Lagrange's method, the structure' shape and finite element unit grids will change in a completely consistent way, and no substance will flow between units. Lagrange's method can be used to accurately describe the complex boundary conditions and the complex material constitutive relationship during high speed collision.

In this paper, the Lagrange's method from LS-DYNA was used.

Material constitutive model. Johnson-Cook's strength model was used to describe the constitutive relationship between projectiles and adjusting pad material [8] as follows: 


$$
\sigma_{y}=\left(A+B \varepsilon_{\mathrm{p}}^{\mathrm{n}}\right)\left(1+C \log \dot{\varepsilon}_{\mathrm{p}}^{*}\right)\left(1-T_{\mathrm{H}}^{\mathrm{m}}\right)
$$

Where $\sigma_{y}$ is the material's yield limit; $A, B, n, C$, and $m$ are the input material-related constants: $A$ is yield stress, $B$ and $n$ reflect the effects of strain hardening; $C$ is a coefficient reflecting strain sensitivity, also called strain sensitivity coefficient; $\varepsilon_{\mathrm{p}}^{\mathrm{n}}$ is equivalent plastic strain; $\dot{\varepsilon}_{\mathrm{p}}^{*}=\dot{\varepsilon}_{\mathrm{p}} / \dot{\varepsilon}_{0}$ is dimensionless strain rate, $\dot{\varepsilon}_{\mathrm{p}}$ is plastic strain rate and $\dot{\varepsilon}_{0}$ is reference strain rate.

$$
T_{\mathrm{H}}=\left(T-T_{\text {room }}\right) /\left(T_{\text {melt }}-T_{\text {room }}\right)
$$

where $T$ is the current temperature, $T_{\text {room }}$ is the reference temperature (generally room temperature, but some scholars think $T_{\text {room }}$ should be the material's lowest temperature when used, or the tested lowest temperature ${ }^{[8]}$ ), and $T_{\text {melt }}$ is the material's melting point (in computation, we suppose there is no heat transfer between units, or namely adiabatic condition).

On the right side of Eq. (1), the first pair of brackets indicate the material's basic yield stress and the effect of strain hardening on yield stress; the second pair of brackets indicate the effect of strain rate; the third pair of brackets indicate the effect from the current temperature; the temperature $T$ should be larger than the reference temperature $T_{\text {room }}$; this part is always smaller than 1 , indicating the test material's thermal softening effect under very high temperature. Equation (1) indicates that when temperature $T$ reaches the material's melting point $T_{\text {melt }}$, the yield stress is 0 .

State equations are expressed as follows: ${ }^{[9-11]}$ :

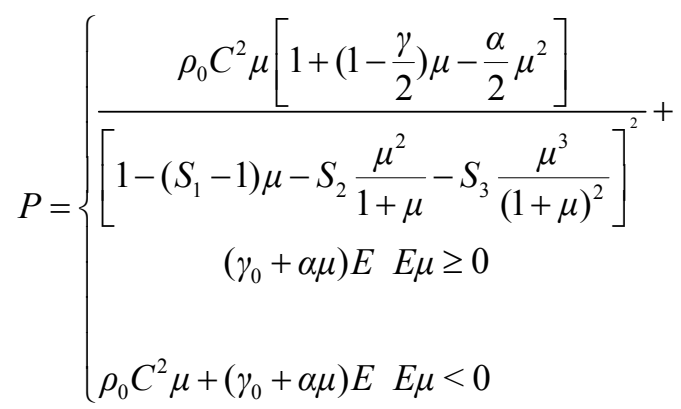

Equations [9-11] indicate that when the material is under tension and compression, different state equations can be used to express the pressure produced by the material. In the above equations, $P$ is pressure; $E$ is the material's specific internal energy; $\rho$ is the current density; $\rho_{0}$ is the initial density; $C$ is the intercept of the material's curve $v_{\mathrm{s}}-v_{\mathrm{p}} ; S_{1}, S_{2}$, and $S_{3}$ are the coefficients of curves $v_{\mathrm{s}}-v_{\mathrm{p}} ; v_{\mathrm{s}}$ is impact wave speed; $v_{\mathrm{p}}$ is particle speed, the two can be approximated as a linear relationship (namely $S_{2}=S_{3}=0$ ); $\mu^{\prime} \rho / \rho_{0}-1$ is compression ratio; $\gamma_{0}$ is a Grüneisen constant; $\alpha$ is a 1-order volume correction coefficient of $\gamma_{0}$.

The projectiles are made of 4340 steel; the adjusting pad materials are 5A06 aluminum, 2A12 aluminum, and 7A04 aluminum. Tables 1 and 2 show the relevant physical parameters of the four

\begin{tabular}{|c|c|c|c|c|c|c|c|c|c|}
\hline s & material & $\left.{ }_{3}\right) \quad \rho(\mathrm{kg} / \mathrm{m}$ & $\mu$ & $E(\mathrm{Gpa}$ & )$^{A(\mathrm{MPa}}$ & $B(\mathrm{MPa}$ & $n$ & $C$ & $m$ \\
\hline el & 4340ste & 7830 & 0.33 & 200 & 792 & 510 & 0.26 & 0.014 & 1.03 \\
\hline $\mathrm{Al}$ & 5A06 & 2700 & 0.33 & 70 & 168.4 & 950.5 & 0.71 & 0.0165 & 1.08 \\
\hline $\mathrm{Al}$ & $2 \mathrm{~A} 12$ & 2770 & 0.33 & 71.7 & 400 & 635 & 0.42 & 0.015 & 1.426 \\
\hline & 7A04 & 2850 & 0.33 & 69.35 & 602.5 & 732.1 & 0.753 & 0.014 & 1.51 \\
\hline
\end{tabular}
materials ${ }^{[12]-[15]}$.

Table 1 Parameters of materials model 


\begin{tabular}{llllll}
\hline materials & $C(\mathrm{~m} / \mathrm{s})$ & $S_{1}$ & $S_{2}$ & $S_{3}$ & $\gamma_{0}$ \\
\hline 4340steel & 4580 & 1.49 & 0 & 0 & 1.93 \\
$5 \mathrm{~A} 06 \mathrm{Al}$ & 5370 & 1.338 & 0 & 0 & 1.67 \\
$2 \mathrm{~A} 12 \mathrm{Al}$ & 5370 & 1.338 & 0 & 0 & 1.97 \\
$7 \mathrm{~A} 04 \mathrm{Al}$ & 5370 & 1.4 & 0 & 0 & 1.99 \\
\hline
\end{tabular}

The Hopkinson bar and the anvil body both maintain the elastic state during working, and thus their material constitutive models both utilize isotropic linear elastic material model. The Hopkinson bar and the anvil body both are made of TC4 titanium alloy, with density of $4505 \mathrm{~kg} / \mathrm{m}^{3}$, elastic modulus of $113 \mathrm{GPa}$, and Poisson's ratio of $0.4^{[16]}$.

Systematic numerical model. To study the effects of projectile nose shape, nose length, and adjustment pad material on the accelerometer's excitation waveforms, we used three nose shapes (Fig. 2); three nose lengths (Fig. 3), and three adjustment pad material (5A06 Al, 2A12 Al, and $7 \mathrm{~A} 04 \mathrm{Al})$.

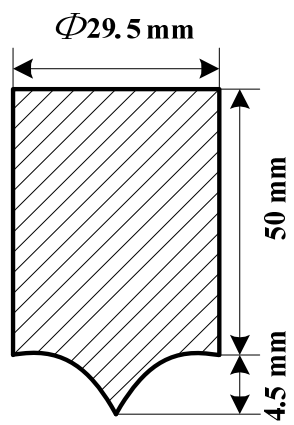

$\# 1$

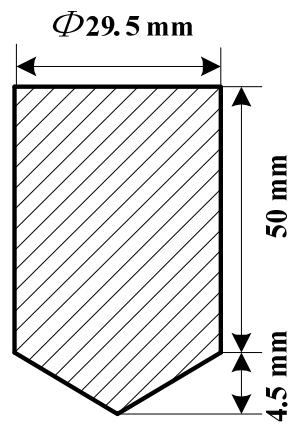

\#2

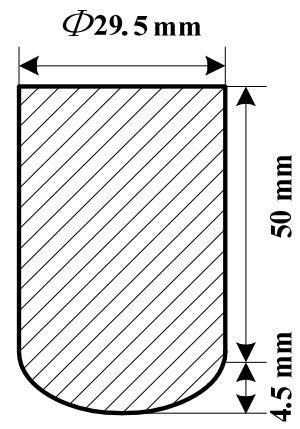

\#3

Fig.2 Sketch of projectiles with different nose shape
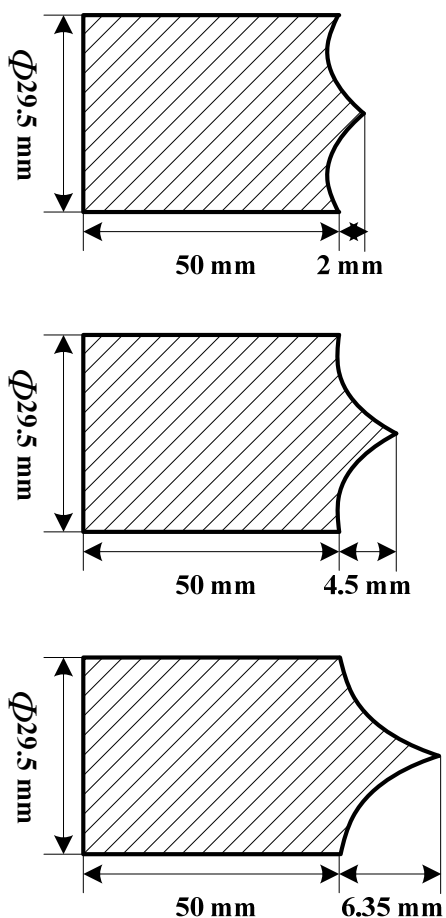

projectiles \#1 with different nose length
\#\#1

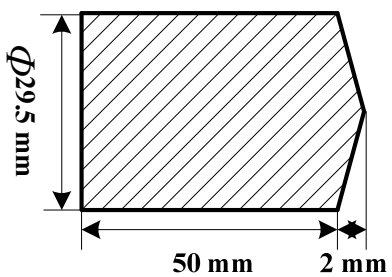

\#\#\#1

\#\#2

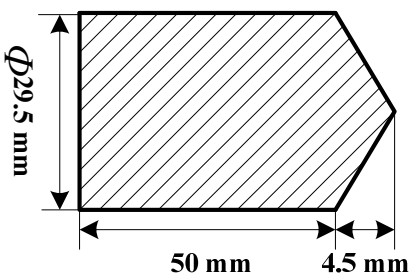

\#\#\#2

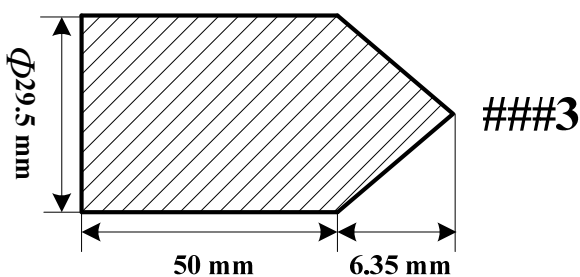

projectiles \#2 with different nose length

Fig.3 Sketch of projectiles with different nose length 
Considering that the system and load are both symmetrical around the Hopkinson bar's central axis, a 2D axisymmetric geometric model was built for the whole system (Fig. 4), including projectiles, adjustment pad, Hopkinson bar, and the anvil body. The finite element grids of systematic impact excitation were locally amplified. The unit type is set as plane 162 axisymmetric unit. The contacts between a projectile and the adjustment pad, between the adjustment pad and the Hopkinson bar, and between the Hopkinson bar and the anvil body are all set as single face automatic contact, with restraining conditions as follows: the vertical displacement of axial nodes is zero and projectile speed is $40 \mathrm{~m} / \mathrm{s}$. To avoid distortion of unit shape and to control the quality of acceleration waveforms, the units near the contact area between projectiles and the adjustment pad were intensified appropriately. The end time of the solving process is decided by the time $t$ when the stress wave is propagated from the bar's one end to the other end. A TC4 Hopkinson bar has a stress wave propagating speed of $5100 \mathrm{~m} / \mathrm{s}^{[16]}$ and bar length of $1.52 \mathrm{~m}$, and thus $\mathrm{t}$ is $\sim 303$. There is certain duration from when the stress wave reflects repeatedly in the anvil body until when the anvil body flies away from the bar, and thus the end time of the solving process is set as 700 .

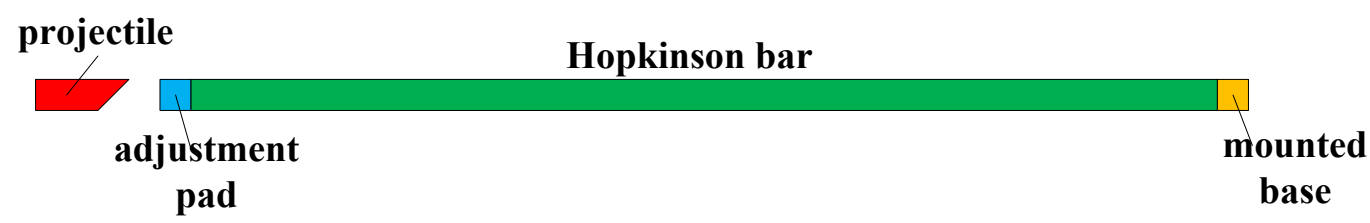

(a) $1 / 2$ geometry model of the high $\mathrm{G}$ accelerometer calibration system
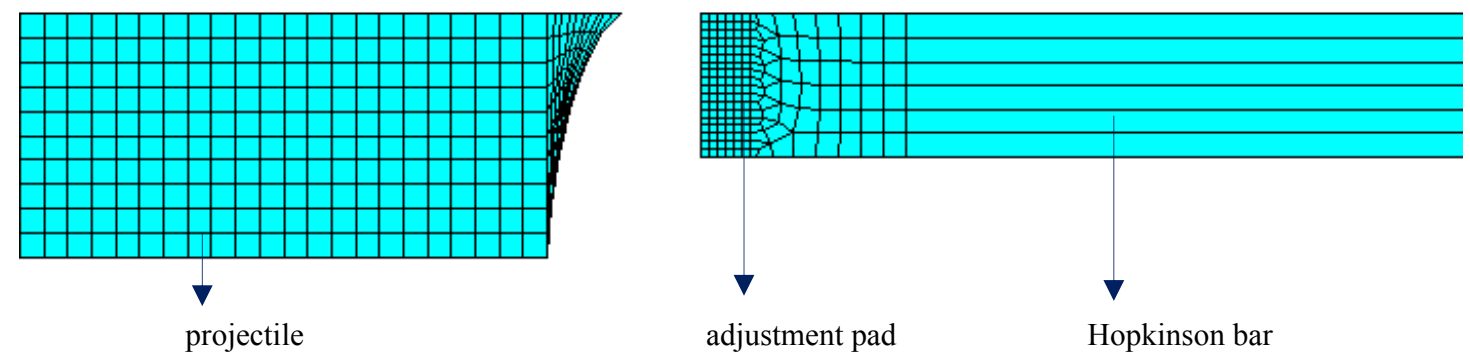

(b) FE model of the shock excitation part

Fig.4 Numerical model of the high $\mathrm{G}$ accelerometer calibration system

\section{Numerical simulated and test results and analysis}

Effects of projectile nose shape on acceleration waveforms. Fig. 5 shows the simulated results and test results of rigid acceleration-time curves of the anvil body, when the projectiles with three nose shapes are impacting the 2A12 aluminum adjustment pad at the speed of $40 \mathrm{~m} / \mathrm{s}$. Clearly, the simulated results and the test results are highly consistent and both show the same changing trend. Under the action of projectile \#1, the anvil body's acceleration pulse has the longest rise time, smallest amplitude, and largest pulse width. From projectile \#1 to projectile \#3, the rise time of the acceleration pulse gradually decreases, the amplitude increases, and the pulse width decreases. 


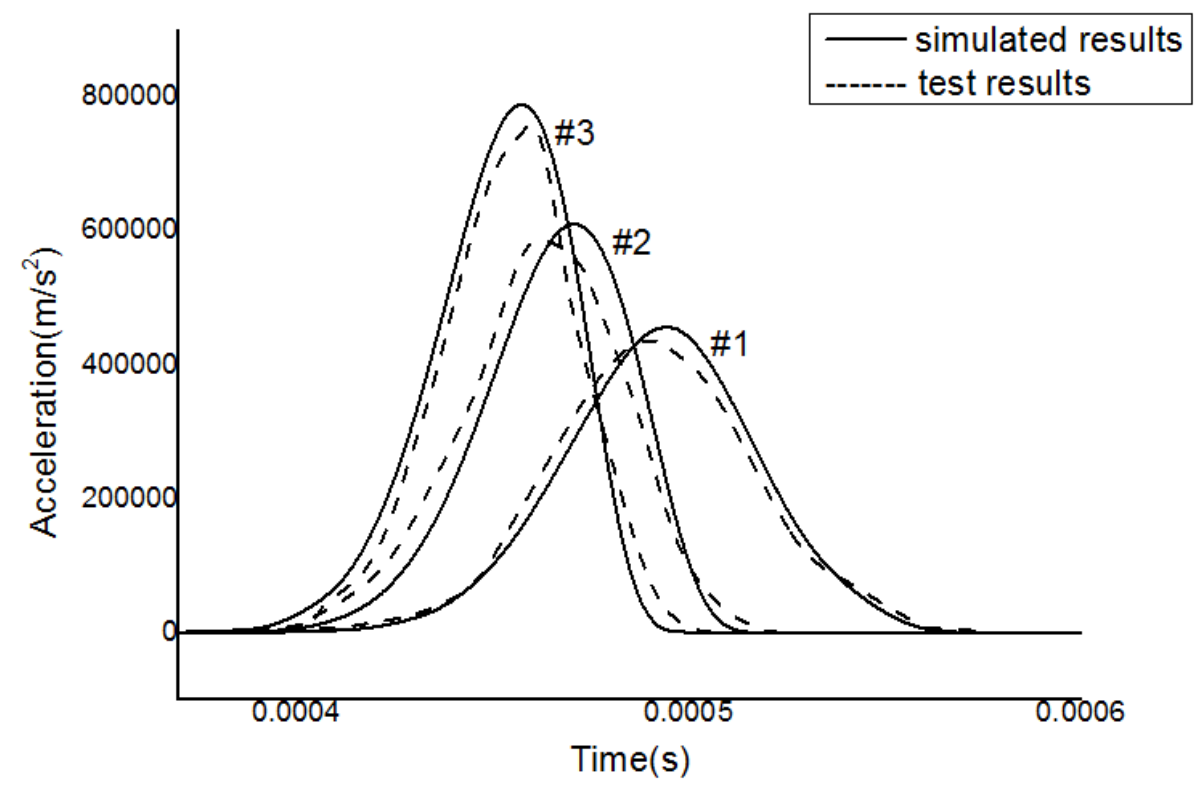

Fig.5 Acceleration curve of projectiles with different nose shape impacting $2 \mathrm{~A} 12$ aluminum pad at $v_{0}=40 \mathrm{~m} / \mathrm{s}$

Effects of projectile nose length on acceleration waveforms. Fig. 6 and 7 show the simulated results and test results of anvil body's rigid acceleration-time curves with nose shape \#1 and \#2 respectively, the projectiles with three nose lengths are impacting the 2A12 Al adjustment pad at the speed of $40 \mathrm{~m} / \mathrm{s}$.

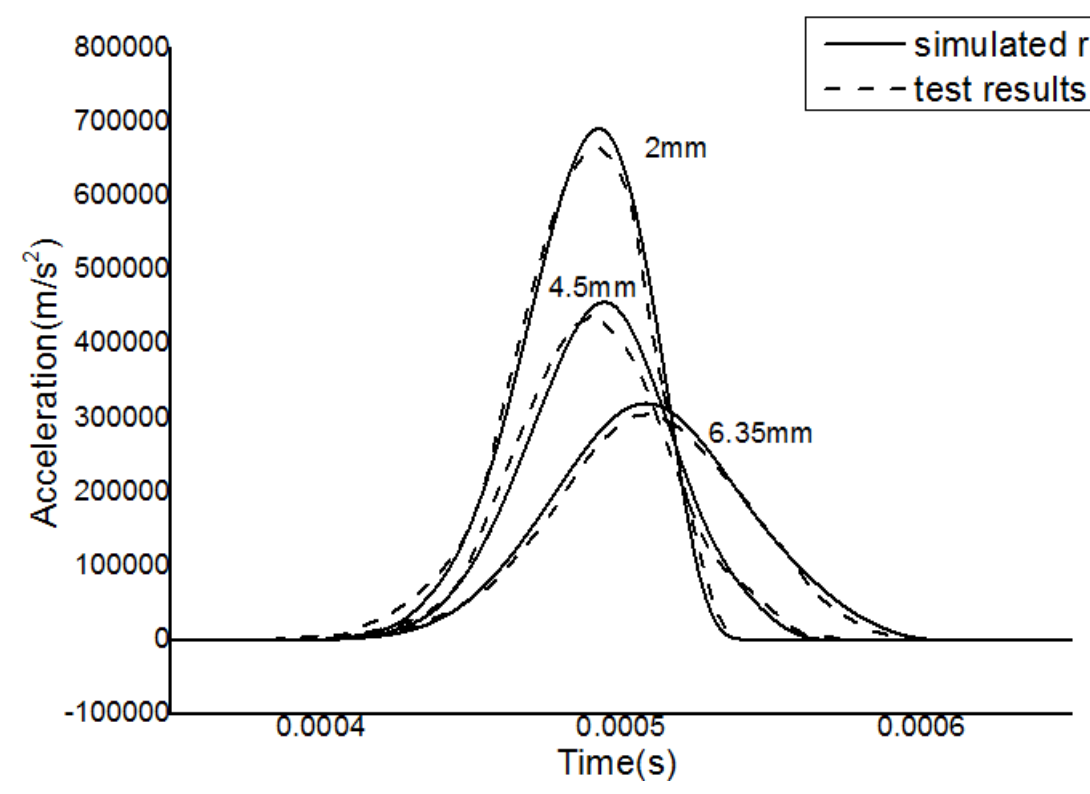

Fig.6 Acceleration curve of projectiles \#1 with different nose length impacting $2 \mathrm{~A} 12$ aluminum pad at $v_{0}=40 \mathrm{~m} / \mathrm{s}$ 


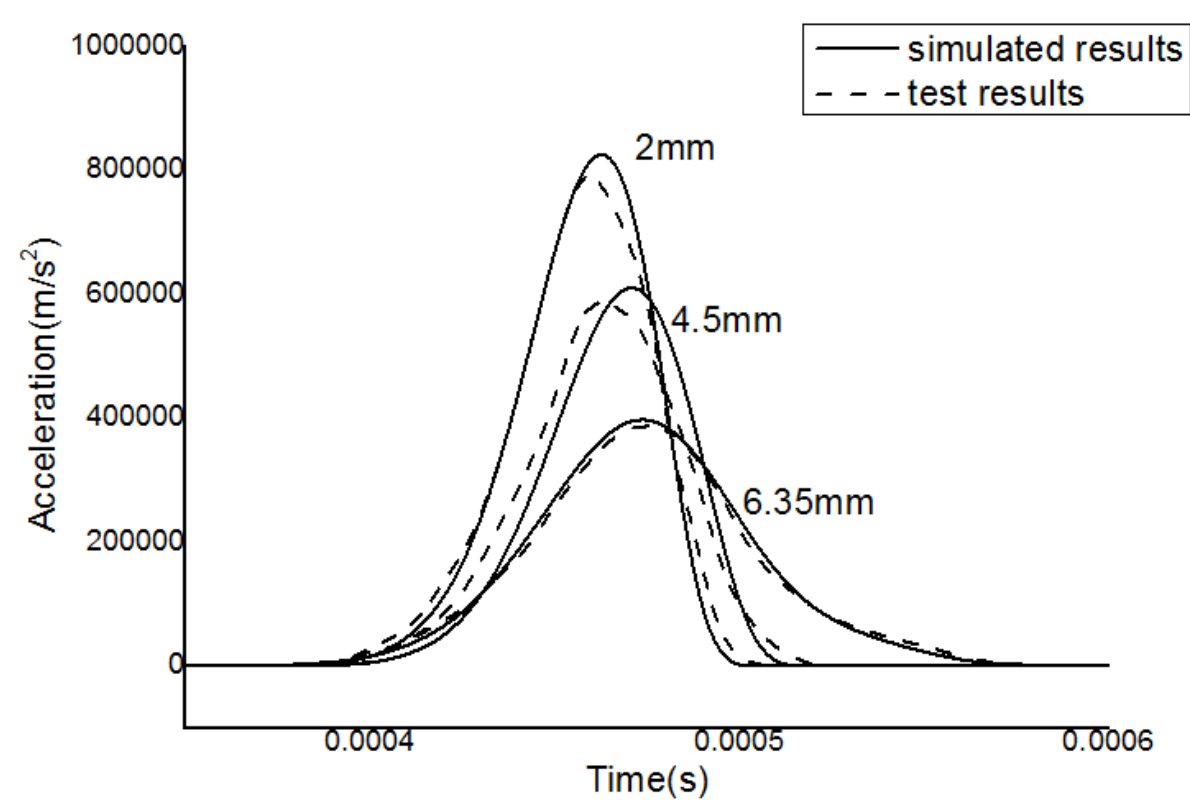

Fig.7 Acceleration curve of projectiles \#2 with different nose length impacting $2 \mathrm{~A} 12$ aluminum pad at $v_{0}=40 \mathrm{~m} / \mathrm{s}$

Clearly, the simulated results and the test results are highly consistent and both reflect the same varying trend. Under the action of projectiles with the nose shape, when the nose length is the smallest, the anvil body's acceleration pulse has the shortest rise time, largest amplitude, and smallest pulse width. Along with the increase of nose length, the acceleration pulse's rise time gradually increases, the amplitude decreases, and the pulse width increases.

Effects of adjusting pad material on acceleration waveforms. Fig. 8 shows the simulated results and test results of anvil body's rigid acceleration-time curves, when \#\#2 projectiles are impacting the $5 \mathrm{~A} 06 \mathrm{Al}, 2 \mathrm{~A} 12 \mathrm{Al}$ and 7A04 Al adjustment pads at the initial speed of $40 \mathrm{~m} / \mathrm{s}$. Clearly, the simulated results and the test results are highly consistent and both reflect the same variation trend. When the projectiles are impacting the 5A06 Al adjustment pad, the anvil body's acceleration pulse has the longest rise time, smallest amplitude, and largest pulse width. From 5A06 Al to 7A04 Al, the acceleration pulse's rise time gradually decreases, the amplitude increases, and the pulse width decreases.

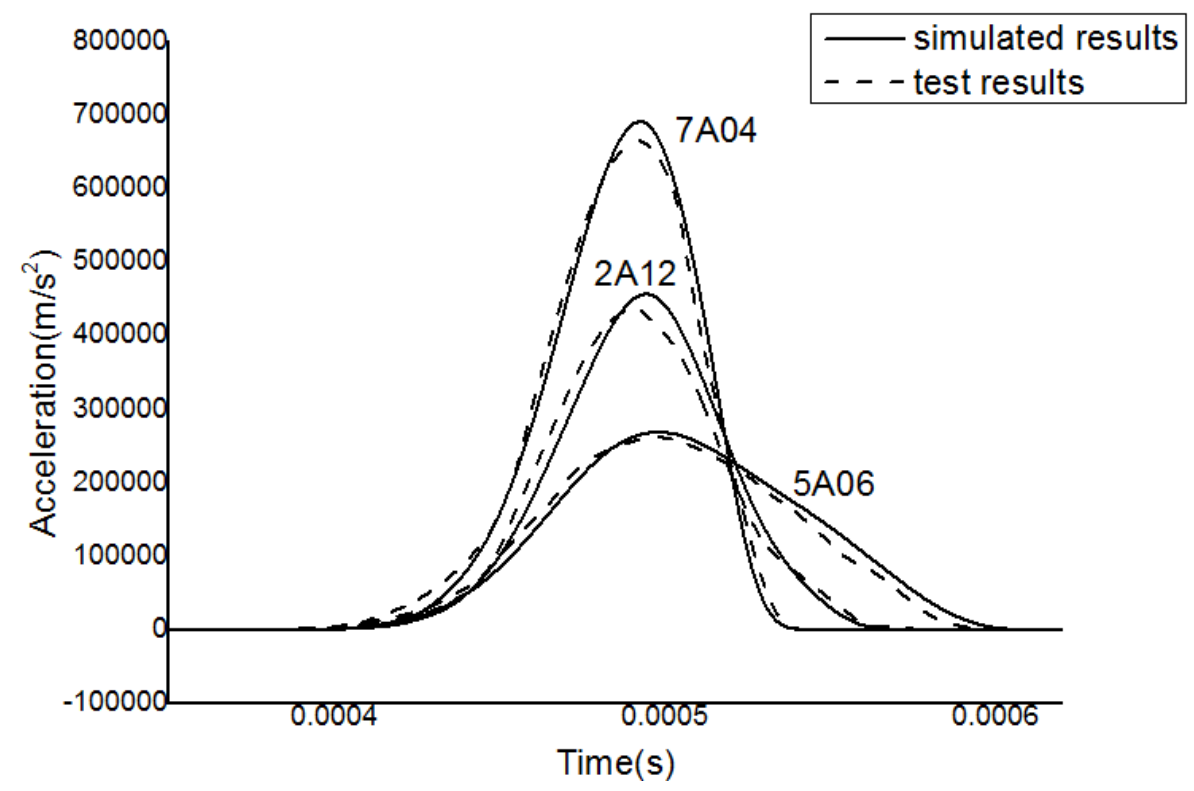

Fig.8 Acceleration curve of projectiles \#\#2 impacting 5A06,2A12 and 7A04 aluminum pad at $v_{0}=40 \mathrm{~m} / \mathrm{s}$ 
Analysis of results. Nose taper gradually decreases when nose shape is changed from \#3 to \#1 (Fig. 2) and when the nose length increases (Fig. 3), and thus the impacting area between a projectile and the adjustment pad gradually decreases. When the projectiles impact the adjustment pad at the same initial speed, the local stress around the impacting point increases, and thus plastic waves with lower speed can be more easily produced in the adjusting pad, and the projectile's kinetic energy will be consumed more by the adjustment pad's plastic deformation. Thereby, the adjustment pad's loading speed will decrease and the loading duration will be prolonged. Therefore, the Hopkinson bar's pressure stress pulse will have longer rise time, and the plastic wave with small local wave speed will be propagated forward; amplitude will decrease with the enlarged area. Because the anvil body's acceleration pulse width approximates the pressure stress wave's front rise time in the Hopkinson bar [16]-[17], the acceleration pulse's rise time will be prolonged, pulse width increases, and amplitude decreases.

Table 2 shows that when the adjusting pad material is changed from 5A06 Al to 7A04 Al, its yield stress gradually increases, and thus when the projectiles impact at the same initial velocity, the adjustment pad with lower yield stress will generate larger plastic deformation and thus consume more kinetic energy. Therefore, it will produce acceleration waveforms with smaller amplitude, longer rise time, and larger pulse width.

\section{Conclusions}

To calibrate the high-G accelerometers using Hopkinson bar test installation, different excitation acceleration waveforms can be obtained by changing projectile nose shape, nose length, or adjusting pad material.

(1) Projectiles with small nose taper or with large nose length will impact and produce excitation acceleration waveforms with small amplitude, long rising time, and large pulse width.

(2) For the adjusting pad material with small yield stress, the projectiles will impact and produce acceleration waveforms with small amplitude, long rise time, and large pulse width.

Based on the results from numerical simulation and experimental validation, the high-G accelerometer calibration system using Hopkinson bar will provide reference values.

\section{References}

[1] Q. Deng, Y.X. Wu, R.Q. Shen, Y.H. Ye. Application of pulse shaper in over-loading test for initiator. Initiators and Pyrotechnics, 2005, 12(5): 12-15.

[2] J.B. Fan, J. Zu, Z.S. Lin, P. Xu, X.D. Zhao. Shock calibration for a high-g accelerometer using a laser interferometer. Journal of Vibration and Shock, 2012, 31(11): 149-153.

[3] ISO 16063-13. Methods for the calibration of vibration and shock transducers[S]. Part 13: Primary Shock calibration using laser interferometry, 2001.

[4] Johnson G R, Cook W H. A constitutive model and data for metals subjected to large strain, high strain rates and high temperatures //Proceedings of 7th International Symposium on Ballistics. Hague: Netherlands, 1983: 541-547.

[5] Johnson G R, Cook W H. Fracture characteristics of three metals subjected to various strains, strain rates, temperatures and pressures. Engineering Fracture Mechanics, 1985, 21(1): 31-48.

[6] Kazunaga Ueda, Akira Umeda. Improvement of novel NRLM method for accelerometer characterization to the range 102ms-2. Sensor and Actuators(A), Vol.54, 2003, 517-522.

[7] Y.L. Li, W.G. Guo, D.X. Jia, F. Xu. An equipment for calibrating high shock acceleration sensors. Explosion and Shock Waves, 1997,17(1): 90-96.

[8] R.Q. Liang. Elastic-plastic constitutive modeling of tantalum and Aermet 100 steel due to quasi-static and dynamic loading. USA: University of Maryland, 1999. 
[9] M.S. Lin, B.J. Pang, W. Zhang, R.Q. Chi. Experimental investigation on a dynamic constitutive relationship of 5A06 Al alloy. Explosion and Shock Waves, 2009, 29(3): 306-311.

[10]M. J. Forrestal, T.C. Togami, W. E. Baker, and D. J. Frew. Performance evaluation of accelerometers used for penetration experiments. Experimental Mechanics, Vol.43, 2003, No.1.90-96.

[11] Urtiew P A, Vandersall K S, Tarver C M, et al. Shock initiation experiments and modeling of Composition B and C-4//Proceedings of the 13th International Detonation Symposium, Norfolk, Virginia, USA, 2006,23-28 July, pp.929-939.

[12]J. Wang, H.G. Cao. EFP penetrating explosive reactive armor. Journal of Nanjing University of Science and Technology, 2008, 32(1): 9-10.

[13] P. Xu, J. Zu, J.B. Fan. Theoretical analysis and numerical simulation of waveform adjustment in accelerometer calibration on Hopkinson bar//The International Symposium on Impact Dynamics. Tiyuan: Shanxi, PRC,2011:176-185.

[14] F.J. Zhang, J.L. Tao, C.J. Tian. Application of cone-cylinder projectiles in split Hopkinson pressure bar test. Journal of Experimental Mechanics, 2003,18(1): 137-140.

[15] W. Zhang, G. Wei, X.K. Xiao. Constitutive relation and fracture criterion of 2A12 aluminum alloy. Introducing Journal of China Ordnance, 2013, 34(3): 276-282.

[16] W. Zhang, X.K. Xiao, G. Wei. Constitutive relation and fracture model of 7A04 aluminum alloy. Explosion and Shock Waves, 2011, 31(1): 81-87.

[17]X.D. Zhao. Theoretical and experimental study on the shock calibration for high-g accelerometers. PRC: North university of China, 2010. 